\title{
THE ENERGY EMISSION OF SHEETS IN THE THE PULSAR MAGNETOSPHERE
}

\author{
TONG YI \\ Beijing Normal University \\ Li ZHONG YUAN \\ University of Science and Technology of China
}

\begin{abstract}
We present a possible emission mechanism based on the idea of current sheets in magnetohydrodynamics. The current sheets are formed close to the light cylinder due to a relativistic effect involving partly frozen-in particles. We estimate that the energy emitted by the current sheets fits the observations fairly well.
\end{abstract}

\section{Introduction}

The pulsar emission problem has received the attention of many astronomers (Michel 1981) and is a very important problem. Some investigators, e.g., Ruderman and Sutherland (1975), believe that the emission originates in the polar-cap gap region close to the star, whereas others (Smith 1977) argue that it is emitted near the velocity-of-light cylinder.

In this paper, we present a possible emission mechanism based on the idea of magnetohydrodynamic current sheets. Because the excited plasma turbulence results in anomalous resistance near the light cylinder of a pulsar, the frozen-in condition is partly relaxed. In addition the magnetic field lines in this area cannot perfectly corotate with the star due to relativistic effects on the partly frozen-in particles. The differentially rotating lines are drawn along backward and therefore lead to the formation of current sheets. The emission comes from such current sheets, which act as an energy source for the curvature or synchrotron radiation, giving rise to the observed emission spectrum.

\section{Parameter and energy estimates}

The mass of particles increases rapidly near the light cylinder of a pulsar due to relativistic effects. Therefore, the magnetic field lines no longer corotate with the star, because the tremendously massive frozen-in particles are too heavy to constrain, even if only partially frozen-in. So the magnetic field lines are expected to lag behind and to be curved in both the fully and partially frozen-in cases (see figure 1), particularly close to the light cylinder.

The curved magnetic field lines will create an induced electromotive force. We may estimate the

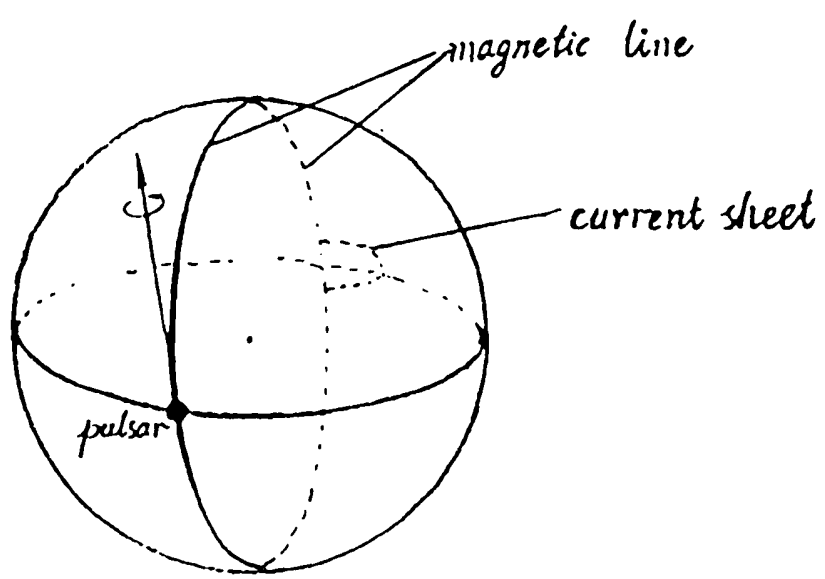

Figure 1 Formation of the current sheet

electric field strength as

$$
\int E \cdot d l=-\frac{1}{c} \int \frac{\partial B}{\partial t} d s .
$$

Approximating the above equation

$$
E \cdot 4 z^{*}=-\frac{1}{c} \frac{\Delta B}{\Delta t} z^{*}
$$

the area $\Lambda$ originally (figure 1) has no magnetic field which are curved now and then penetrate this place, so $\Delta P=B$. We introduce a parameter $\xi$ to describe the extent of the frozen-in condition, which is between zero and unity, and will be defined later. The magnetic annihilation time $\tau_{\mathrm{D}}=z^{*} V_{\text {in }}$ is the speed of magnetic field lines going into the diffusion and $z^{*}$ is the width of that region. Assuming this is a dipolar magnetic field, $B$ can be expressed as $B \doteq B_{0} R^{3} / 2 \tau^{3}$, where $R_{0}$ is the radius of a pulsar, and $r$ is the distance from the center of the star. Presumably it is reasonable that $B$ should be multiplied by $\xi$ because only those field lines with frozenin particles lag behind and induce the electric field. Replacing $\Delta t$ by $\tau_{\mathrm{D}}$, we obtain $E \approx(\xi B / 4 c) V_{\text {in }}$. 
Since angular momentum is conserved for a system without a moment of force, we have the relation $m V r=\gamma_{\text {in }} m V^{\prime} r$. The left side of the equation represents the angular momentum of a particle with mass $m$, located at $r$ from the center of the pulsar, and with revolution velocity $V$, whereas the right side includes the relativistic effect on the particle and correspondingly the revolution velocity becomes $V^{\prime \prime}$ (where $\gamma_{\text {in }}$ is the Lorentz factor). Then we obtain the velocity delay $\Delta V=V^{\prime}=V\left(1-1 / \gamma_{\text {in }}\right)$; here $V=\Omega r$, and $\Omega$ is the angular velocity of the pulsar. We rewrite this equation in the form $\Delta V=\Omega r\left(1-1 / \gamma_{\text {in }}\right)$ which in equilibrium gives a result close to the value of $V$ mentioned above.

For describing the frozen-in extent more quantitatively we introduce a parameter $\xi$ which is defined as $\xi=e^{-1 / R_{\mathrm{m}}}$, where $R_{\mathrm{m}}$ is the Reynolds' number. Obviously $\xi \rightarrow 1$ when the conductivity tends to infinity, corresponding to fully frozen-in case, and $\xi \rightarrow 0$ when $\sigma$ tends to zero corresponding to a diffusion case; $\xi \sim 0.37$ corresponds to the currentsheet case in which $R_{\mathrm{m}} \sim 1$.

Using $V_{\text {in }} \approx \Omega \gamma\left(1-1 / \gamma_{\text {in }}\right)$, we can rewrite the equation describing the electric field strength as

$$
E \approx-\frac{\xi B}{4 c} \Omega \gamma\left(1-1 / \gamma_{\text {in }}\right)
$$

So far we do not know whether the plasma is turbulent and thus whether there is interaction between the charged particles and the plasmons. Considering only the collisions between the ions and electrons, the conductivity $\sigma_{\mathrm{c}}$ of the plasma has the form of $\sigma_{\mathrm{c}} \approx n e^{2 / m_{\mathrm{e}} \nu_{\mathrm{ei}}}$ for the strong magnetic field case, where $n$ is the number density of electrons, $e$ and $m_{\mathrm{e}}$ the electric charge and mass of the electron, respectively, and $\nu_{\mathrm{ei}}$ the collision frequency. The expression for $\nu_{\mathrm{ei}}$ has the form $\nu_{\mathrm{ei}} \approx \omega_{\mathrm{pe}} / N_{\mathrm{D}}$, where $\omega_{\mathrm{pe}}$ is the frequency of an electron plasma and $N_{\mathrm{D}}$ the Debye number which is related to the Debye length $d_{\mathrm{e}}$ and equal to $n_{\mathrm{e}} d_{\mathrm{e}}^{3}$. In the relativistic case

$$
E \approx-\frac{\xi B}{4 c} \Omega r\left(1-\frac{1}{\gamma_{\text {in }}}\right)
$$

(Kaplan and Tsytovich 1973), and we take $\xi=2.5$ and obtain $\sigma_{\mathrm{c}} \approx 6.9 \times 10^{6} \gamma^{1 / 2}\left(T_{\mathrm{e}} K^{3}\right)^{3 / 2}$ where $E_{\mathrm{e}}=$ $\gamma m_{\mathrm{e}} C^{2}$, and $T_{\mathrm{e}}$ is the electron temperature in units of Kelvin temperature.

The drift velocity of the electrons relative to the ions is $V_{\mathrm{d}}=\sigma_{\mathrm{c}} E / n e$, which only includes the particle collisions. Substituting eq.(1) in the expression for $V_{\alpha}$ and taking $n \sim 10^{10} \mathrm{~cm}^{-3}$ and $\Omega \sim 10^{2} \mathrm{rad} \mathrm{s}^{-1}$, it is fairly easy to find $V_{\mathrm{d}}>c / \sqrt{3}$, which is an acoustic velocity. $V_{d}$ will never be greater than the speed of light, because $\sigma_{\mathrm{c}}$ decreases due to anomalous resistance as soon as $V_{\mathrm{d}}$ is greater than $c / \sqrt{3}$. So we expect that either an ion-acoustic wave or Buneman (1959) instability arises, producing plasma turbulence and thus anomalous resistance. This is true even at $r=0.9 R_{\mathrm{c}}$, where $R_{\mathrm{c}}$ is the radius of the light cylinder.

The anomalous conductivity in a strong magnetic field may be expressed as $\sigma_{\mathrm{A}} \approx c v_{\mathrm{P}}^{2} / 4 \pi \omega_{\mathrm{H}}$ (Kaplan and Tsytovich 1973), where $\omega_{\mathrm{H}}=e B c / \varepsilon$ and assuming only electrons and ions involved $\omega_{\mathrm{p}}^{2}=$ $\sum_{\alpha} \omega_{\mathrm{px}}^{2} \approx \omega_{\mathrm{pe}}^{2}$. Substituting $\omega_{\mathrm{H}}$ and $\omega_{\mathrm{p}}^{2}$ into $\sigma_{\mathrm{A}}$, we obtain the anomalous conductivity

$$
\sigma_{\mathrm{A}} \approx 72 \frac{n}{B}
$$

Since the conductivity is finite near the light cylinder of a pulsar, the frozen-in condition may be partially relaxed. The field lines are curved near the light cylinder and form current sheets. For convenience we take the shape of the sheets to be rectangular with length $\chi^{*}$ and width $z^{*}$. For this particular case the mass-conservation equation has the form

$$
V_{\text {in }} \chi^{*}=V_{\text {out }} z^{*}
$$

on the basis of the mechanism proposed by Petschek and Thorne (1967); and using the calculation method of Vasylinuas (1975), we obtain the same equation as they did

$$
\left(z^{*}\right)^{2}-\lambda z^{*}-\lambda_{e}^{2}=0,
$$

where the resistance length $\lambda=c^{2} / 2 \pi \sigma V_{\text {in }}$ and the electron inertial length $\lambda_{\mathrm{e}}=c \sqrt{m_{\mathrm{e}} / 2 \pi n e^{2}}$, except for the trivial differences in the coefficients. Obviously $\lambda \gg \lambda_{e}$ holds in the case we are considering, and the width $z^{*}$ of the diffusion region is

$$
z^{*} \approx c^{2} /[2 \pi \sigma \omega r(1-1 / \gamma)] .
$$

If we take $n \sim 10^{10}$ as before, we find that the width of the diffusion region is roughly $z^{*} \sim 10^{5} \mathrm{~cm}$. By use of eq.(3) when $\lambda \gg \lambda_{e}$, the length $\chi^{*}$ of the diffusion region is as follows $\chi^{*} \approx c^{2} / 4 \pi \sigma M V_{\text {in }}$, where $M$ is the magnetic merging rate $M=$ $V_{\text {in }} / V_{\text {out }}$. If the magnetic field in the reconnection region and the kinetic relevant to $V_{\text {in }}$ outside the sheet comparatively are negligible, we have $n \gamma_{\text {out }} m_{\mathrm{ec}}^{2}=(\xi B)^{2} / 8 \pi$. Then we obtain $\gamma_{\text {out }}=$ $4.8 \times 10^{4}\left[(\xi B)^{2} / n\right]$, that is $V_{\text {out }}=c\left(1-1 / \gamma_{\text {out }}^{2}\right)^{1 / 2}$, where $c$ is the light speed. The magnetic merging rate then becomes

$$
M=\frac{\Omega \gamma\left(1-1 / \gamma_{\text {in }}\right)}{c\left(1-1 / \gamma_{\text {out }}^{2}\right)^{1 / 2}} .
$$

It is likely that the emitted energy of a pulsar results from the transfer of the magnetic field whose energy comes from the rotating pulsar. Also we see that the magnetic energy is sufficient to 
supply the radiation. The estimate of the annihilation time $\tau_{\mathrm{D}}$ is about $10^{-5} \mathrm{~s}$. The power supplied by the magnetic field through a current sheet is about $(8 \pi)^{-1}(\xi B)^{2} / \tau_{\mathrm{D}}$, which represents about $1.36 \times 10^{14} \mathrm{erg} \mathrm{cm}^{-1} \mathrm{~s}^{-1}$ for $B \approx 5 \times 10^{5}$ Gauss near the light cylinder, $\xi$ being roughly equal to 0.37 in the current sheet. Assume that the emission region is located near the equatorial plane with outer radius $R_{c}$ and inner radius $0.9 R_{c}$, so the total emission from a volume $0.2 \pi z^{*} R_{\mathrm{c}}^{2}$ is about $8.5 \times 10^{34} \mathrm{erg} \mathrm{s}^{-1}$, which agrees fairly well with the observational value - e.g., the case of PSR 0531+21.

The charged particles gain energy through the magnetic merging and are then captured by the magnetic field outside the field reversal region. These particles release and distribute their kinetic energy in various ways such as curvature and/or synchrotron radiation.

\section{Conclusions}

1. Because the electron drift velocity is greater than the acoustic velocity near the light cylinder of a pulsar, either an ion-wave or Buneman instability occurs, which results in the appearance of anomalous resistivity.

2. Since the conductivity of the plasma is not infinite, the frozen-in state is necessarily partially relaxed. This is the actual precondition to create a current sheet.

3. Some particles frozen-in to the magnetic field near the light cylinder cannot corotate with the central pulsar due to relativistic effects and thus cause the field lines to lag forming current sheets.

4. If the mechanism presented in this paper is correct, the number density of charged particles in the Goldreich and Julian (1969) model is low in the radiation region, because the ejected particle velocity appears to be unreasonable. 